\title{
Bionic design and performance test of maize grain cleaning screen through earthworm motion characteristics
}

\author{
Lijun Wang ${ }^{*}$, Yongtao Yu, Shuai Zhang, Xin Feng, Lianglai Song \\ (College of Engineering, Northeast Agricultural University, Harbin 150030, China)
}

\begin{abstract}
The maize mixture feeding with a large mass cannot be migrated backward rapidly along the planar reciprocating vibrating screen, and it is easy to accumulate in the front of the screen, which leads to the decrease of screening efficiency. Based on the reverse engineering technology, using the wavy geometry formed during the earthworm (Pheretima guillelmi) moving as a bionic prototype, a bionic screen was designed to make the maize mixture migrate backward rapidly in the front of the screen. The contour curve of earthworm's head in an axial contracted state was extracted and fitted to obtain its equation. Based on the difference of concave position of the lower surface's wavy geometry during the earthworm moving, the motion of the bionic screen was divided into four postures, and the conversion between different postures of the bionic screen was realized by the cam drive mechanism. The kinematics simulation of the bionic screen was carried out through ADAMS, and the displacement and velocity of the bionic screen were analyzed. When the feeding mass of the maize mixture was set at 5 $\mathrm{kg} / \mathrm{s}, 6 \mathrm{~kg} / \mathrm{s}$ and $7 \mathrm{~kg} / \mathrm{s}$, the test results showed that the time of the maize mixture migrated (TOMMM) in the front of the bionic screen was shortened by $0.18 \mathrm{~s}, 0.71 \mathrm{~s}$, and $1.36 \mathrm{~s}$, respectively, compared with that of planar reciprocating vibrating screen. The total screening time (TST) of the bionic screen was shortened by $1.28 \mathrm{~s}, 1.33 \mathrm{~s}$, and $1.53 \mathrm{~s}$, respectively. The ability of the maize mixture to be migrated backward was improved. This study can provide a reference for the innovative design of the cleaning screen.
\end{abstract}

Keywords: cleaning screen, maize grain harvester, material accumulation, earthworm, bionic design, motion characteristics DOI: $10.25165 /$ j.ijabe.20211403.6534

Citation: Wang L J, Yu Y T, Zhang S, Feng X, Song L L. Bionic design and performance test of maize grain cleaning screen through earthworm motion characteristics. Int J Agric \& Biol Eng, 2021; 14(3): 12-21.

\section{Introduction}

The functions of maize grain harvesters which are becoming the main development direction of maize harvesters in China, include ear-picking, peeling, threshing, and cleaning ${ }^{[1]}$. The cleaning device is a critical component of maize grain harvesters. The cleaning of the maize mixture including grains, stalks, cobs, and a small number of lightweight impurities is the last procedure before maize grains enter the grain box ${ }^{[2,3]}$. However, after the maize mixture leaves the threshing device, it will first fall onto the oscillation grain pan at the inlet of the cleaning device and then fall onto the front of the screen. Therefore, the maize mixture is easy to accumulate in the front of the screen ${ }^{[4,5]}$. As the working width of the combine harvester and the yield of crops per unit continues to increase year by year, the feeding mass continues to be increased $^{[6]}$. The maize mixture could not be migrated backward rapidly in the front of the planar reciprocating vibrating screen, making the accumulation of the maize mixture more serious, resulting in a reduction in screening efficiency and the operating efficiency of the maize grain harvester ${ }^{[7]}$.

\section{Received date: 2021-02-18 Accepted date: 2021-04-18}

Biographies: Yongtao Yu, PhD candidate, research interest: cleaning technology and bionic design, Email: yyt2015110104@163.com; Shuai Zhang, PhD candidate, research interest: cleaning technology and bionic design, Email: zhxmzhangshuai@163.com; Xin Feng, PhD, research interest: grain harvesting machine, Email: fx2020fx@163.com; Lianglai Song, Master candidate, research interest: grain cleaning device innovative design, Email: songlianglai@163.com. *Corresponding author: Lijun Wang, $\mathrm{PhD}$, Professor, research interest: grain harvesting machine. College of Engineering, Northeast Agricultural University, Harbin 150030, Heilongjiang, China. Tel: +86-451-55191897, Email: wanglijun@neau.edu.cn.
To solve the maize mixture's accumulation in the front of the cleaning screen, researchers have performed numerous studies from different aspects. Regarding the working parameters of the screen, Dong et al. ${ }^{[8]}$ simulated the particle flow of a bend/low head screen combination in coal preparation. The horizontal velocity of particles increased by increasing the horizontal amplitude, and the accumulation of particles could be reduced. Zhao et al. ${ }^{[9]}$ analyzed the effects of vibration parameters on the circularly screening processes. With the increase of the vibration frequency, the accumulation of particles at the feeding end decreased. Ma et al. ${ }^{[10]}$ investigated the influences of the turning angle of the cleaning screen's front swing bar on the particles' motion using the discrete element method (DEM). The results showed that the turning angle of the front swing bar had a significant effect on the horizontal dispersion of particles. Regarding the structure of the screen, Liu et al. ${ }^{[1]}$ studied the effects of the inclination angle of the screen on the screening process of the banana screen by using DEM. The results showed that increasing the inclination angle of the front of the screen could reduce the accumulation of materials. Deng et al. ${ }^{[12]}$ designed a non-planar pit screen and a non-planar wave screen, which increased the effective screening area and decreased the accumulation of materials on the screen. Regarding the driving mechanism of the screen, Modrzewski et al. ${ }^{[13]}$ designed a double-frequency screen driven by two inertial drive vibrators operating at various rotation speeds, which could avoid the accumulation of particles in the center of the screen surface. Jiang et al. $^{[14]}$ proposed a variable-amplitude screen with a larger amplitude at the feed end than that at the discharge end, and the migration velocity of particles on the screen was effectively improved. Wang et al. ${ }^{[15]}$ compared the dispersion of particles on a planar reciprocating vibration screen, a vibration screen with 
three translations and one swing, and a vibration screen with three translations and two rotations. The results showed that the multi-dimensional motion was more beneficial for the dispersion of particles on the vibrating plate than the planar motion did.

The horizontal velocity of the material along the screen surface increased by changing the working parameters of the screen. However, a too fast horizontal velocity would affect grains to traverse the openings of the screen. The increase of the inclination angle of the front of the screen reduced the accumulation of materials. However, the materials on the screen could not be migrated backward rapidly under a large feeding mass. The innovative design of the driving mechanism enabled the screen to achieve multi-dimensional motion. However, the further increase of the material migration velocity on the screen was limited because of the fixed shape of the screen surface during the motion.

Scholars have paid more and more attention to solve the problems in agricultural machinery through the principle of bionics. Studies have shown that the performance of agricultural machinery designed through bionics has been effectively improved ${ }^{[16,17]}$. Ma et al. ${ }^{[18]}$ designed a bionic non-smooth vibrating screen based on the characteristics of adhesion and resistance reduction of the bionic non-smooth surface and the function of hydrophobic self-cleaning of the biological surface, which revealed the mechanism of adhesion and resistance reduction of the cleaning screen surface. Cheng et al. ${ }^{[19]}$ modified a cleaning screen surface with polytetrafluoroethylene and designed a cleaning screen surface of the vibrating screen with the performance of adhesion and resistance reduction to reduce the friction factor and interface adhesion force between the rice mixture and the screen surface.

The main objective of this study was to design and test a bionic screen for maize grain harvester to make the maize mixture migrate backward rapidly in the front of the cleaning screen, so as to avoid material accumulation and improve the screening efficiency of the cleaning screen.

Therefore, a bionic screen based on the motion characteristics of earthworms was designed to make the maize mixture migrate backward rapidly and improve the screening efficiency of the cleaning screen under the large feeding mass. It is of great significance to improve the operating efficiency of maize grain combine harvester. In this paper, earthworms were used as bionic prototypes. The contour curve of the earthworm's head in an axial contracted state was extracted using reverse engineering technology, and the maximum concave depth of the bionic screen was determined. The motion posture of the bionic screen was determined based on the difference of concave position of the wavy geometry of the lower surface during the earthworm moving, and the conversion between different postures of the bionic screen was realized by designing the cam drive mechanism. The design of the cleaning screen based on the bionic earthworm motion characteristics was completed. The kinematics simulation of the bionic screen was carried out to verify the rationality of the bionic screen design. Performance tests were carried out with different feeding mass of the maize mixture to verify the maize mixture's ability to migrate backward in the front of the bionic screen and the overall screening efficiency of the bionic screen and the planar reciprocating vibrating screen.

\section{Characteristics of earthworm motion}

\subsection{Analysis of earthworm motion}

The body length of the mature Pheretima guillelmi is 90-
$250 \mathrm{~mm}$, and the body width is $5-10 \mathrm{~mm}$. Because of its large size, it is easy to be observed and measured. Pheretima guillelmi is composed of many similar somites. The circular muscle is located in the outer layer of the body. The longitudinal muscle is located in the inner layer of the body. The body surface is covered with bristles, as shown in Figure 1.

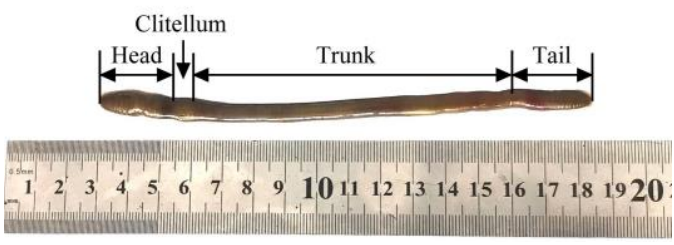

Figure 1 Pheretima guillelmi for the experiment

The earthworm motion is accomplished by the contraction and relaxation of muscles and the assistance of bristles. When the earthworm wriggles forward, the bristles at the back of the body nail into the soil; at this time, the circular muscles contract, the longitudinal muscles relax, then the body stretches forward. Then the bristles at the front of the body nail into the soil; at this time, the longitudinal muscles contract and the circular muscles relax, then the body contracts forward. The backward wave is generated by the muscles that contract and relax alternately, which is opposite to the motion direction of the earthworm and is periodically transmitted along the body, pushing the earthworm to wriggle forward ${ }^{[20,21]}$. The motion process is shown in Figure 2. The wavy geometry surface formed during the earthworm moving is periodically transmitted backward with the earthworm motion. This feature provides inspiration for the maize mixture to be migrated backward rapidly in the front of the cleaning screen. Therefore, the earthworm is selected as the biological prototype for the design of the bionic screen.

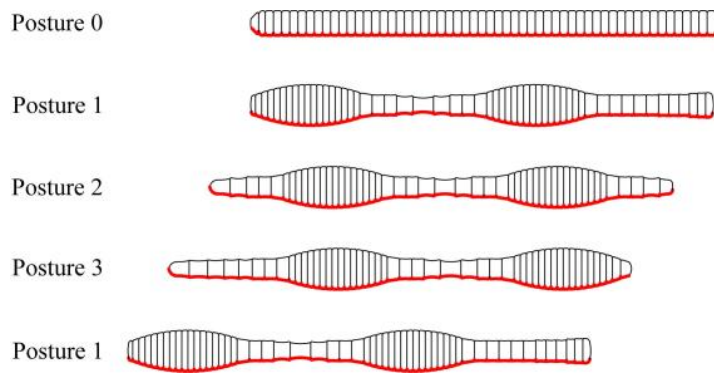

Figure 2 Schematic diagram of earthworm motion

According to the difference in the concave position of the wavy geometry on the lower surface during the earthworm moving, the motion of the earthworm was divided into four postures, as shown in Figure 2, which were named posture 0 , posture 1, posture 2 , and posture 3 , respectively. The posture 0 : the whole body of the earthworm was resting. Posture 1: the earthworm's head and the trunk's middle and rear ends were in an axial contracted state. Posture 2: the earthworm's head and the trunk's middle and rear ends were in the stage of transition from the axial contracted state to the diastolic state. Because this process took a long time, it was divided into a posture alone. Posture 3: the earthworm's head and the trunk's middle and rear ends were in an axial diastolic state.

\subsection{Extraction of the characteristic of earthworm head in an axial contracted state}

There are two contractions at the front and rear during the earthworm moving, and the changes of the height of the earthworm's somites caused by contraction are not significantly different. To reduce the workload of extracting the contour curve of the earthworm and improve image processing accuracy, the 
changes of the height of the earthworm's somites caused by contraction at different positions are regarded as the same. Because the head of the earthworm is easy to be observed ${ }^{[22]}$, the contour curve of the earthworm's head in an axial contracted state is extracted to obtain the maximum height of somites during the earthworm moving. The maximum variation of the height of the earthworm's head somites during moving is obtained to determine the contour curve of the earthworm's body surface.

\subsubsection{Extraction of contour curve of earthworm head}

Earthworms move irregularly in the soil, which is difficult to be observed and photographed. The mature Pheretima guillelmi obtained from earthworm farms (Zhejiang Province, China) was placed on a flat plate to extract the contour curve of earthworms body when moving on a plane. The images of earthworm's head in an axial contracted state were taken by the camera (DSC-RX1RM2, Sony, Japan) to extract their contour curve, as shown in Figure 3.

$2 \mathrm{~mm}$

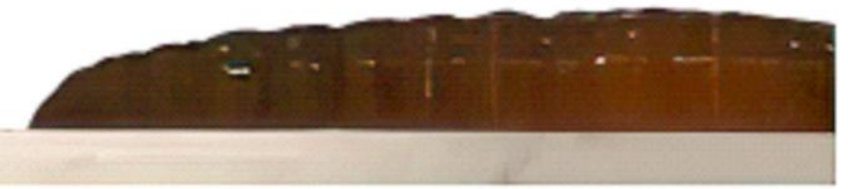

Figure 3 Image of earthworm's head in an axial contracted state

The wavy geometry surface formed during earthworm moving is reflected in the contour curve of earthworm's upper surface. Therefore, by using the image processing technology in computer vision, the contour curves of the upper surface of earthworm's head in the image were extracted by the function command in MATLAB 2014 (Matrix Laboratory, Math Works, USA) software ${ }^{[23,24]}$. The contour curve of the upper surface extracted by the Canny operator was compared with that of the earthworm's original image, as shown in Figure 4. The extracted contour curve was basically consistent with that of the original image. The results showed that the contour curve of the earthworm could be accurately extracted by the above method.

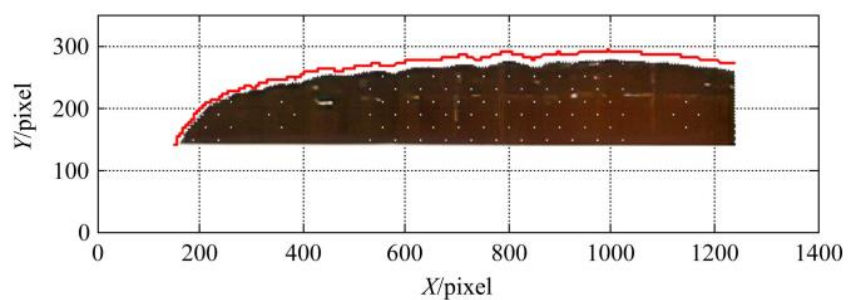

Figure 4 Contour curve of the earthworm's head in an axial contracted state extracted by MATLAB

\subsubsection{Fitting of contour curve of earthworm head}

The curve equation of the contour curve was fitted by MATLAB 2014 to quantitatively analyze the contour curve of the head of Pheretima guillelmi in an axial contracted state ${ }^{[25]}$. The highest-order fitting results of three non-linear curve functions including Gaussian function, polynomial function, and Fourier function were compared. The $R^{2}$ of the eighth-order Fourier function was the highest at 0.9968 , and the SSE was the lowest at 1.098, thus the fitting was the most accurate, as shown in Equation (1):

$$
f(x)=\frac{a_{0}}{2}+\sum_{n=1}^{8}\left[a_{n} \cos (n \omega x)+b_{n} \sin (n \omega x)\right]
$$

2.2.3 Maximum variation of the height of earthworm's head somite

Ten earthworms were randomly selected from the Pheretima guillelmi test samples with a total weight of $0.5 \mathrm{~kg}$. The derivatives of the fitted equations were obtained, respectively, and $f^{\prime}(x)=0$. The maximum value of the function was the maximum height of the earthworm's head somite in an axial contracted state. Then the earthworms were put into the reagent bottles of No. 1-10, respectively, and the ether in the bottles made them unconscious, thus they were at rest. The diameter and length of the head somite were measured with a Vernier caliper. The difference between the maximum height of the earthworm's head somite in an axial contracted state and the height of the somite in an axial resting state is the maximum variation of the height of the earthworm's head somite, which is $3.48 \mathrm{~mm}$, as shown in Table 1 .

Table 1 Physical characteristics of earthworms

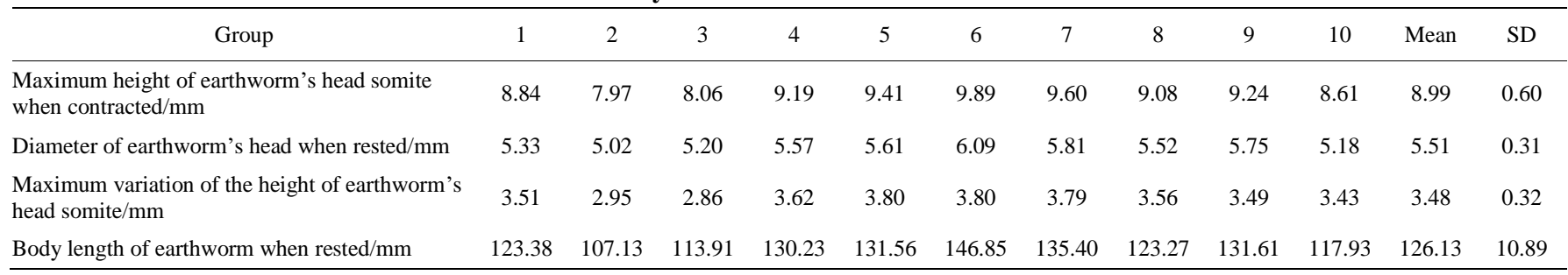

Note: SD = Standard deviation.

\section{Bionic design of cleaning screen}

\subsection{Overall structure and working principle of the bionic} screen

\subsubsection{Overall structure of the bionic screen}

The structure of the bionic screen is shown in Figure 5, which mainly consists of a frame, cam drive mechanism, and screen plate. The side plates are fixed to the front and rear sides of the frame symmetrically, and the driveshaft is fixed on the side plates through the bearing seat. Cam drive mechanisms are installed at the front and rear ends of the driveshaft, respectively, and the screen plate is driven by the pin shaft hinged with the cam driving mechanism. Baffles are installed on both sides of the bionic screen surface. The overlapping parts of adjacent baffles are not in the same vertical plane, thus the baffles and the screen plates would not interfere with each other when the screen is concave. The bionic screen is composed of 16 independent screen plates. From left to right, the screen plate is denoted as 1 to 16 , respectively.

The connection between the combined screens of each group is shown in Figure 6. The combined screen formed by screen plate 1 and screen plate 2 was taken as an example. The sleeves and the pin shafts were fixed on the left and right sides of screen plate 1 and screen plate 2 , respectively. The stretching motion between screen plate 1 and screen plate 2 was achieved by cooperating the sleeves and the pin shafts.

\subsubsection{Working principle of bionic screen}

During the operation, the fan is installed at the front of the bionic screen. The maize mixture is initially stratified and dispersed by the fan's airflow during the process of falling from the oscillation grain pan. The maize mixture is fed to the front of the 
bionic screen, then gathers at the lowest point of the bionic screen surface, and gradually migrates backward with the bionic screen surface to avoid accumulation. The follower is driven by the rotating cam to move up and down. Simultaneously, the combinations of the screen plate are driven by the followers to realize continuous conversion between concave bending and flattening. The maize mixture falling on the bionic screen surface is thrown up, shaken, and loosened so that the impurities and the grains are gradually stratified and dispersed on the screen surface. The maize mixture that has not been cleaned is thrown to the screen plates 9-16, and the above motion is repeated. The separation of maize grains and impurities is completed by the fan's airflow and the motion of the bionic screen.

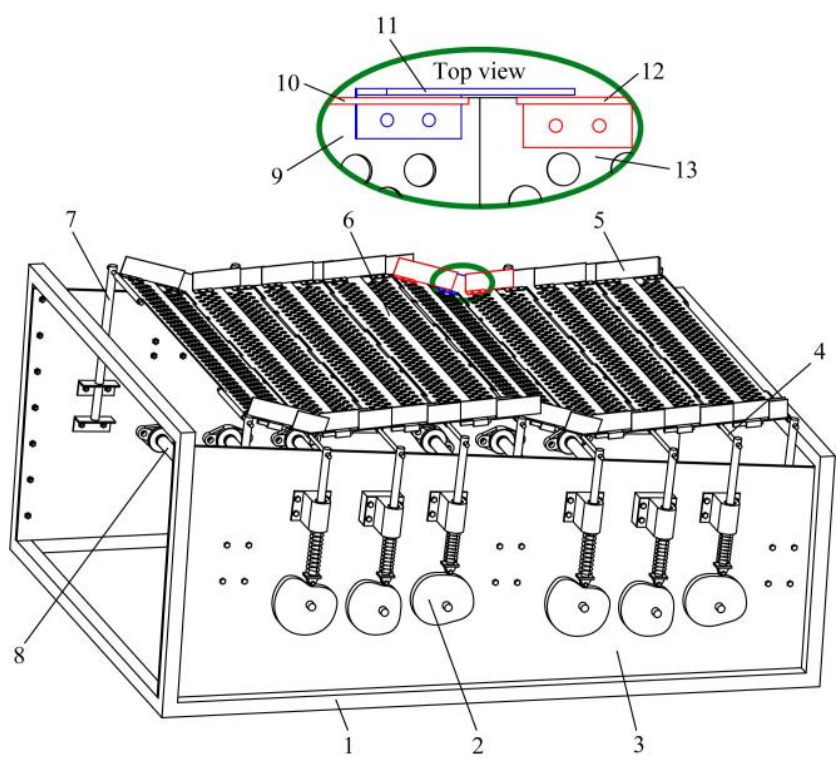

1. Frame 2. Cam drive mechanism 3. Side plate 4. Pin shaft 5. Baffle 6. Screen plate 7. Support rod 8. Driveshaft 9. Screen plate 10 10. Back baffle 9 11. Back baffle 10 12. Back baffle 11 13. Screen plate 11

Figure 5 Schematic diagram of the whole structure of the bionic screen
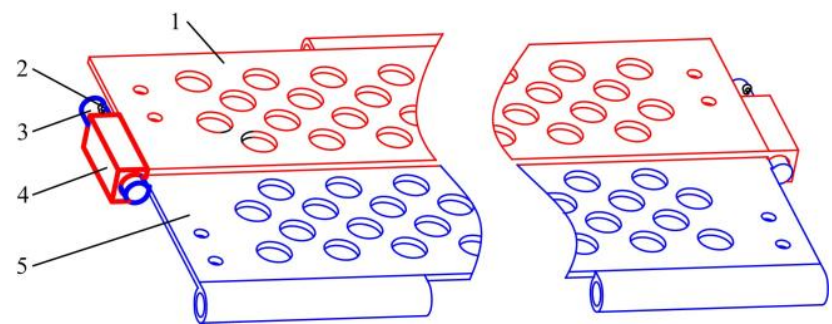

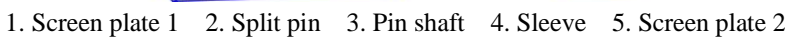

Figure 6 Connection method of the combined screen

\subsection{Determination of the motion posture of bionic screen}

If the bionic screen is convex during maize cleaning, the maize mixture is fed to the front end of the bionic screen, and is blocked by the convex screen plate, then the maize mixture would accumulate and fall directly into the bottom of the screen box from the front end of the bionic screen, as shown in Figure 7. This would affect the screening efficiency and the cleaning rate of maize grain. Therefore, the wave geometry of the lower surface during the earthworm moving was used as the basis of bionics. The bionic screen is converted between concave bending and flattening. When the earthworm moves on a non-hard surface, the contour curves of the upper and lower surfaces are similar. Therefore, the maximum variation of the height of earthworm's head somites obtained from the upper surface contour curve was considered as the maximum concave depth of the lower surface contour curve.

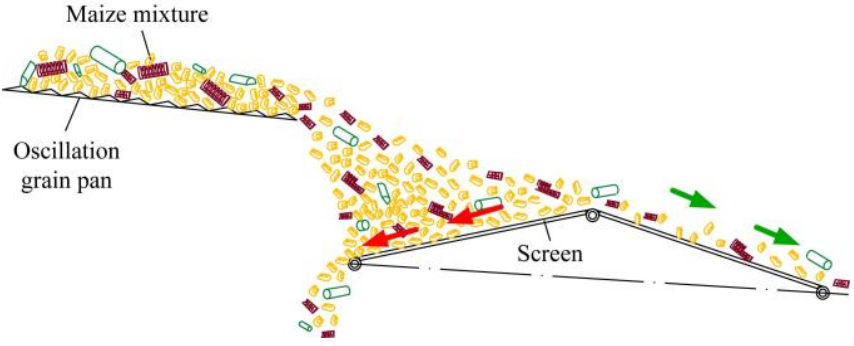

Figure 7 Schematic diagram of the motion of the maize mixture when the bionic screen is convex

The bionic screen motion was divided into four postures, which was posture $0-3$, and the bionic design of the cleaning screen was conducted. The shapes of the wave geometry of earthworm's lower surface were simplified to facilitate the manufacturing. While maintaining the bionic features, the arc contours were straightened $^{[26]}$, and the concave depths of different positions were the same. The motion posture of the bionic screen is shown in Figure 8. Posture 0 is the initial state of the bionic screen. When the bionic screen is in posture 1 , the screen surface in the front of the screen is concave and reaches the lowest point, and the screen surface becomes "V-shaped". As the bionic screen moves from posture 1 to posture 2, the lowest point of the $\mathrm{V}$-shaped screen surface gradually moves to the middle of the screen. When the bionic screen moves to posture 3, the lowest point of the V-shaped screen surface moves from the middle to the rear of the screen, forming a backward wave.

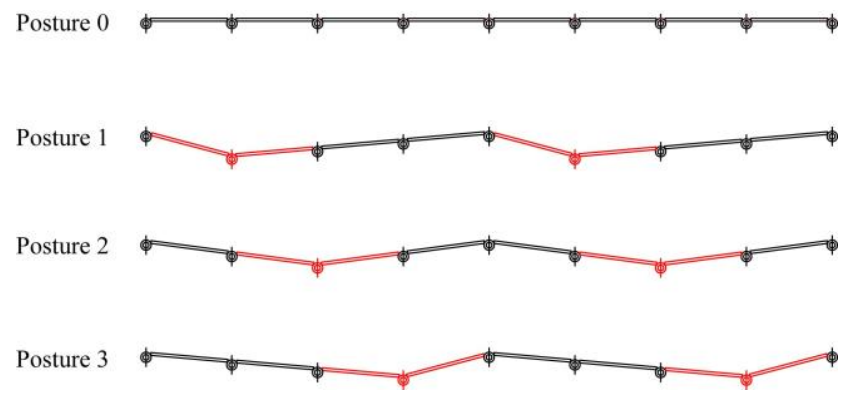

Figure 8 Motion posture of the bionic screen

\subsection{Design of driving mechanism}

The driving mechanism composed of cams and followers was designed to realize the continuous motion of concave bending and flattening of the bionic screen. The drive mechanism of the bionic screen includes 6 pairs of cams, driving all screen plates to realize the conversion between different postures of the bionic screen.

3.3.1 Determination of the maximum concave depth of the bionic screen

The maximum concave depth of the bionic screen $(A)$ was calculated as follows:

$$
A=L \frac{H}{l}
$$

where, $L$ is the total length of the bionic screen, $1500 \mathrm{~mm} ; H$ is the maximum concave depth of lower surface's contour curve of earthworm, $3.48 \mathrm{~mm}$; $l$ is the length of the earthworm, $126.13 \mathrm{~mm}$.

The maximum concave depth of the bionic screen calculated by Equation (2) was $41.39 \mathrm{~mm}$. To improve the stratification and dispersion effect of maize mixture on bionic screen, the concave depth of the bionic screen was appropriately increased and rounded. Therefore, the maximum concave depth of the bionic screen was $50 \mathrm{~mm}$.

\subsubsection{Design of contour curve of the cam}

The followers hinged with the bionic screen were pushed by 
the cams to move up and down when the bionic screen was working. The roller followers were selected to reduce the friction between the cams and the followers. To reduce the rigid impulse between cam and follower, the sine acceleration curve was adopted to the followers ${ }^{[27,28]}$.

The displacement equation of follower in actuating travel is as follows:

$$
s=h\left[\left(\delta / \delta_{0}\right)-\sin \left(2 \pi \delta / \delta_{0}\right) /(2 \pi)\right]
$$

The displacement equation of follower in return travel is as follows:

$$
s=h\left[1-\left(\delta / \delta_{0}^{\prime}\right)+\sin \left(2 \pi \delta / \delta_{0}^{\prime}\right) /(2 \pi)\right]
$$

where, $s$ is the displacement of follower, $\mathrm{mm} ; h$ is the stroke of follower, $\mathrm{mm} ; \delta$ is the motion angle of the cam, $\left({ }^{\circ}\right) ; \delta_{0}$ is the motion angle for actuating travel, $\left({ }^{\circ}\right) ; \delta_{0}{ }^{\prime}$ is the motion angle for return travel, $\left({ }^{\circ}\right)$.

To ensure the normal operation of the cam, its maximum pressure angle $\left(\alpha_{\max }\right)$ should be less than the allowable pressure angle $([\alpha])$ to avoid self-locking. The cam's pressure angle $(\alpha)$ is calculated as follows:

$$
\alpha=\arctan \left|\frac{\mathrm{d} s / \mathrm{d} \delta}{r_{0}+s}\right|
$$

where, $r_{0}$ is the base circle radius of the cam, $50 \mathrm{~mm}$.

The motion angles of travel 1, travel 2, travel 3, and travel 4 of the optimized cam were $50^{\circ}, 90^{\circ}, 70^{\circ}$, and $150^{\circ}$, respectively. At this time, the maximum pressure angles of cam 1, cam 2, and cam 3 were $22.0^{\circ}, 20.3^{\circ}$, and $23.0^{\circ}$, respectively, which were less than the allowable pressure angle $\left([\alpha]=30^{\circ}\right)$, thus there was no self-locking problem. The motion curves and physical figures of cam 1, cam 2 , and cam 3 are shown in Figure 9.

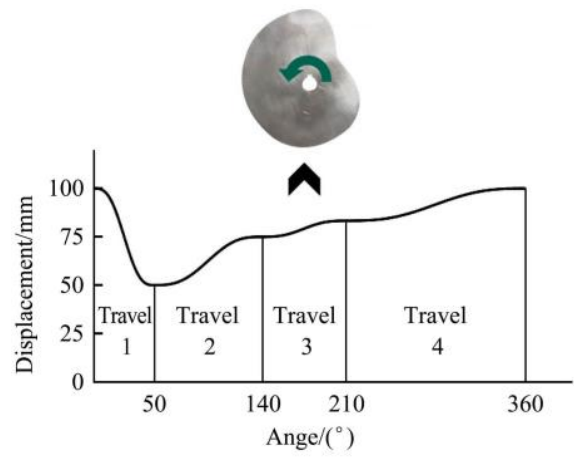

a. Cam 1

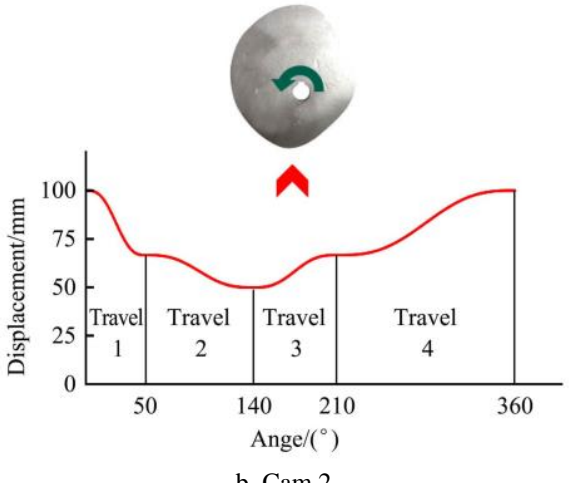

b. Cam 2

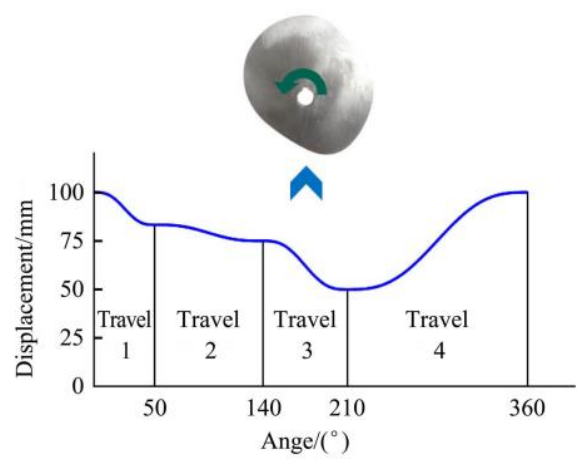

c. Cam 3

Figure 9 Cams and their motion curve

\subsubsection{Reliability analysis of the cam}

To prove the reliability of the bionic screen, the reliability of the cam driving the motion of the bionic screen has been analyzed based on the stress-strength interference model. Suppose the stress $S$ and the strength $\delta$ of the cam obey the normal distribution, namely $S \sim N\left(\mu_{S}, \sigma_{S}^{2}\right), \delta \sim N\left(\mu_{\delta}, \sigma_{\delta}^{2}\right)$, and they are independent of each other. The reliability $(R)$ of the cam was calculated as follows:

$$
R=1-F=\Phi\left(\frac{\mu_{\delta}-\mu_{S}}{\sqrt{\sigma_{\delta}^{2}+\sigma_{S}^{2}}}\right)
$$

where, $F$ is the failure probability of the cam; $\mu_{\delta}$ is the mean value of strength, $\mathrm{MPa} ; \sigma_{\delta}$ is the standard deviation of strength, MPa; $\mu_{s}$ is the mean value of stress, MPa; $\sigma_{s}$ is the standard deviation of stress, $\mathrm{MPa} ; \Phi$ is the value of the integral function of the random variable with standard normal distribution.

The material of cam is $40 \mathrm{Cr}$, and the heat treatment method is quenching and then tempering to 45-50HRC. The mean value $\mu_{\delta}$ and standard deviation $\sigma_{\delta}$ of the cam strength is $785.00 \mathrm{MPa}$ and $43.84 \mathrm{MPa}^{[29]}$, respectively. The mean value $\mu_{S}$ and standard deviation $\sigma_{S}$ of the stress on the cam at different rotational speeds were obtained by ADAMS 2017 (Automatic Dynamic Analysis of Mechanical Systems, MSC, USA) simulation. The reliability of cam 1, cam 2, and cam 3 was obtained by Equation (6), as shown in Figure 10.

As shown in Figure 10, when the rotational speed of cam was from $90 \mathrm{r} / \mathrm{min}$ to $150 \mathrm{r} / \mathrm{min}$, the reliability of cam 1 , cam 2 , and cam 3 was all greater than 0.99 , thus they have high reliability. When the rotational speed of the cam was $90 \mathrm{r} / \mathrm{min}$, the reliability of cam 1 was the highest, which was 0.9996242 . When the rotational speed of the cam was $150 \mathrm{r} / \mathrm{min}$, the reliability of cam 2 was the least, which was 0.99058 . With the increase of the rotational speed, the reliability of cam 1 decreased gradually, the reliability of cam 2 first increased and then decreased, and the reliability of cam 3 changed in a wave shape. When the reliability of the bionic screen is high, the maintenance frequency and cost could be reduced.

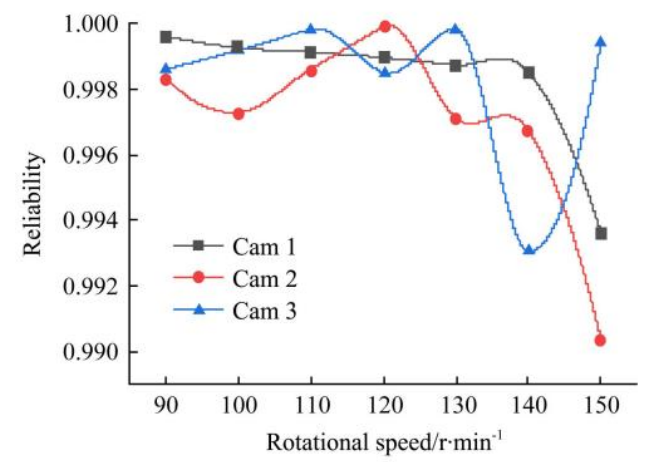

Figure 10 Variation curve of reliability for cam 1, cam 2, and cam 3 with different rotational speeds

\subsection{Design of basic parameters of the bionic screen}

As shown in Figure 11, the range of the distance $E$ between adjacent hinge points was 187.5 to $194.1 \mathrm{~mm}$. To ensure no gap between two independent screen plates when they were stretched to both sides, a certain amount of overlap $j$ was needed after inserting and assembling two-screen plates. Therefore, the length of each screen plate $e$ was $95 \mathrm{~mm}$ and $8 \mathrm{~mm} \leq j \leq 14.5 \mathrm{~mm}$. To ensure that the opening rate of the bionic screen would not be reduced due to a certain amount of overlap between adjacent screen plates, the diameter of the circular hole remains unchanged, while the horizontal and vertical spacing between adjacent circular holes of the bionic screen was reduced by $3.75 \mathrm{~mm}$ and $2.00 \mathrm{~mm}$, 
respectively. The opening rate of the bionic screen was $32.45 \%$, which was $0.14 \%$ higher than that of the planar circular hole screen, thus the performance of the bionic screen was not affected by the opening rate. The gap $u$ between the overlapped parts was $1.5 \mathrm{~mm}$, which avoided the friction during the moving of the two-screen plates and also ensured that the maize grain could not enter the overlapping part. The inclination angle of the whole installation of the bionic screen surface was $-3.5^{\circ}$.

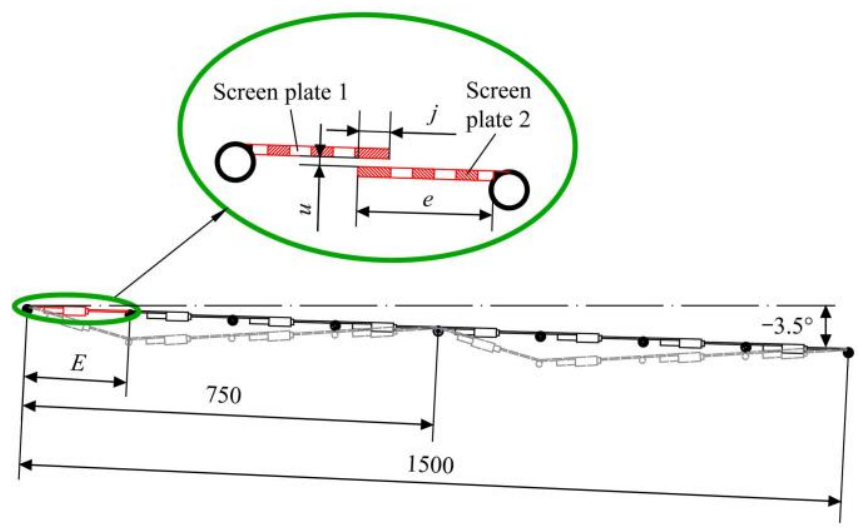

Figure 11 Schematic diagram of the design of bionic screen structure

\section{Analyses of the characteristics of the bionic screen moving}

The 3D model of the bionic screen was built by using Pro/E 5.0 (Pro/Engineer, PTC, USA) and imported into ADAMS 2017. The material properties, constraints, forces, and drives of the bionic screen model were set ${ }^{[30]}$. The rotational speed of the cams was set to $90 \mathrm{r} / \mathrm{min}$. The simulation model of the bionic screen in ADAMS is shown in Figure 12.

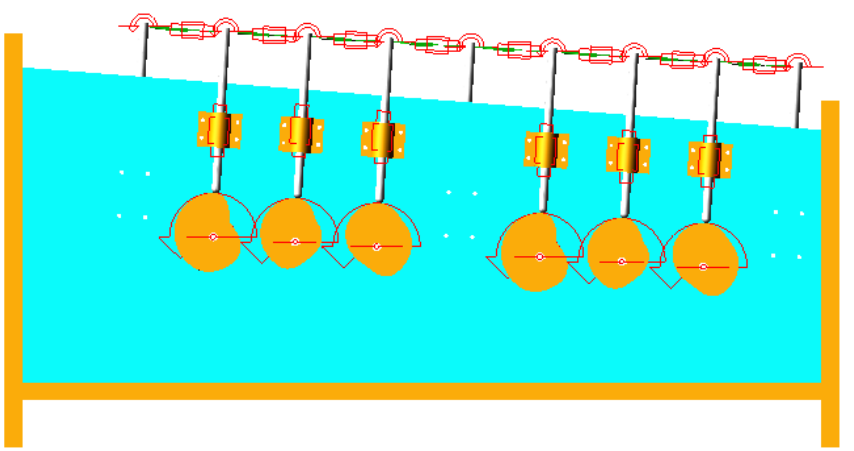

Figure 12 Simulation model of the bionic screen in ADAMS

\subsection{Displacement of screen plates}

The screen plates 1-8 and 9-16 of the bionic screen are two groups of identical screen plates, and their motions are also the same. Therefore, only the motions of screen plates 1-8 were analyzed. The displacements of screen plates 1-8's geometric center over time were shown in Figure 13. The displacement of each screen plate was non-linear, decreasing first and then increasing with time in the same motion period. The screen plates were first concaved, and when the maximum concave depth was reached, the screen plates started to move upward to be flattened. The displacement of the screen plates near the feeding end reached maximum earlier than that at the discharge end. The displacement variation of screen plates 1 and 8 was the smallest, which was $15.83 \mathrm{~mm}$, and the displacement variation of screen plates 3 and 6 was the largest, which was $45.55 \mathrm{~mm}$.

The displacements of screen plates 1-8's geometric centers at
$0 \mathrm{~s}, 0.093 \mathrm{~s}, 0.260 \mathrm{~s}$, and $0.389 \mathrm{~s}$ are shown in Figure 14. It was consistent with posture 0 , posture 1 , posture 2 , and posture 3 of the actual motion state at the same time. The maximum concave depth of the bionic screen formed in the motion process gradually moved backward with time, which was beneficial for maize mixture to be migrated backward. The results showed that the requirements of the motion posture of the screen during the bionic screen moving and the mutual conversion between different postures could be realized by the cooperation of cam drive mechanisms.

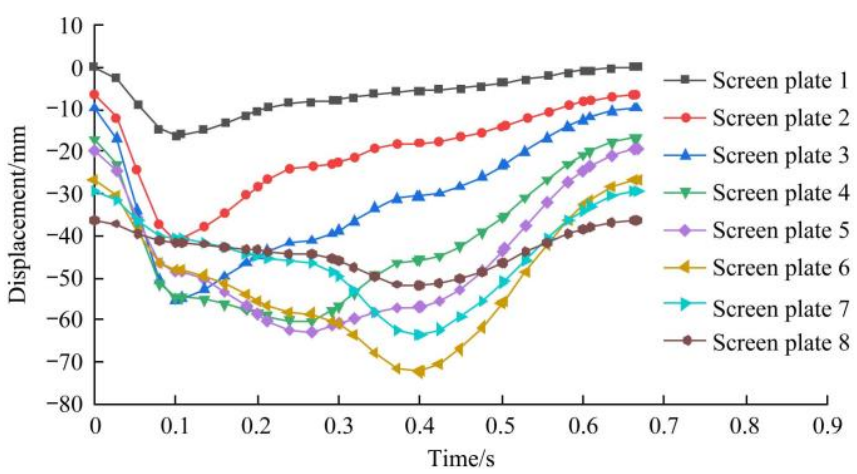

Figure 13 Displacement curve of the screen plates 1-8

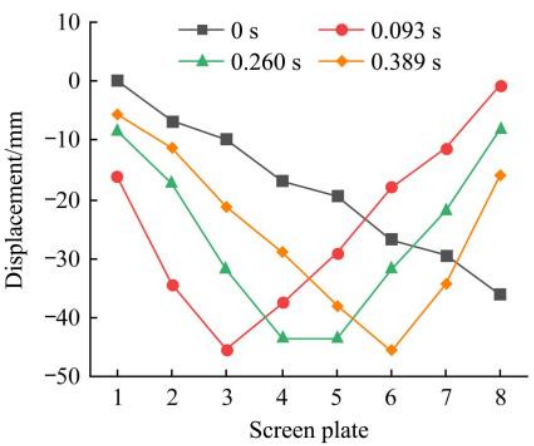

Figure 14 Displacement of screen plates 1-8 at different times

\subsection{Velocity of screen plates}

The velocity curves of screen plates 1-8 in the vertical direction are shown in Figure 15. The velocity curve of each screen plate was composed of four small arc curves in a motion period corresponding to four motion travels of the cam. The velocity is negative when the screen plates move downward.

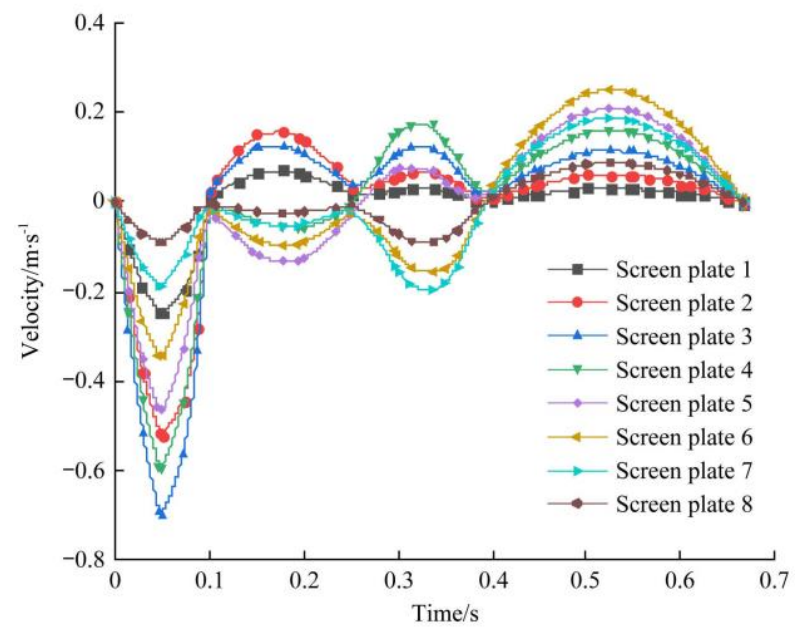

Figure 15 Velocity curve of the screen plates 1-8 in the vertical direction

It could be seen from Figure 15 that screen plates 1-8 all moved downward in the first motion travel of the cam, and the 
bionic screen moved from posture 0 to posture 1 . The screen plates 1, 2, and 3 moved upwards in the second motion travel of the cam, while the rest screen plates continued to move downwards, and the bionic screen moved from posture 1 to posture 2. Except for screen plates 6,7 , and 8 , the other screen plates moved upward in the third motion travel of the cam, and the bionic screen moved from posture 2 to posture 3 . All the screen plates moved upward in the fourth movement of the cam, and the bionic screen moved from posture 3 to posture 0 . The above motion was repeated to complete the screening of the grains and the separation of impurities.

\section{Laboratory tests on bionic screen performance}

\subsection{Materials and methods}

\subsubsection{Materials}

The main maize variety Xianda 205 grown in the third accumulated temperate zone in the northeast region of China was chosen for the tests. The maize grains, maize stalks, maize cobs, and lightweight impurities were uniformly mixed according to the proportion of each component in the maize mixture obtained during the field tests with a maize grain combine harvester. The proportions are shown in Table 2.

Table 2 Moisture contents and percentages of each component in maize mixture

\begin{tabular}{|c|c|c|c|c|}
\hline Component & $\begin{array}{l}\text { Moisture } \\
\text { content } / \%\end{array}$ & Category & $\begin{array}{c}\text { Percentage } \\
1 \%\end{array}$ & $\begin{array}{c}\text { Total } \\
\text { percentage } / \%\end{array}$ \\
\hline \multirow{3}{*}{ Maize grain } & \multirow{3}{*}{$27.91 \pm 0.86$} & Cone & $54.6 \pm 1.58$ & \multirow{3}{*}{$73.3 \pm 2.13$} \\
\hline & & Spherical & $11.1 \pm 0.56$ & \\
\hline & & Rectangle & $7.6 \pm 0.38$ & \\
\hline \multirow{4}{*}{ Maize stalk } & \multirow{4}{*}{$37.62 \pm 1.93$} & $28 \mathrm{~mm}^{[\mathrm{a}]}$ cylinder & $2.4 \pm 0.19$ & \multirow{4}{*}{$17.6 \pm 0.38$} \\
\hline & & $36 \mathrm{~mm}$ cylinder & $5.7 \pm 0.43$ & \\
\hline & & $44 \mathrm{~mm}$ cylinder & $5.3 \pm 0.35$ & \\
\hline & & $52 \mathrm{~mm}$ cylinder & $4.2 \pm 0.31$ & \\
\hline \multirow{3}{*}{ Maize cob } & \multirow{3}{*}{$57.09 \pm 2.18$} & $1 / 4$ cylinder & $4.4 \pm 0.40$ & \multirow{3}{*}{$8.7 \pm 0.25$} \\
\hline & & $1 / 2$ cylinder & $1.8 \pm 0.15$ & \\
\hline & & Whole cylinder & $2.5 \pm 0.07$ & \\
\hline $\begin{array}{l}\text { Lightweight } \\
\text { impurity }\end{array}$ & $68.31 \pm 5.68$ & & $0.4 \pm 0.03$ & $0.4 \pm 0.05$ \\
\hline
\end{tabular}

Note: ${ }^{[\mathrm{a}]} 28 \mathrm{~mm}, 36 \mathrm{~mm}, 44 \mathrm{~mm}$, and $52 \mathrm{~mm}$ are the length of the cylinder.

\subsubsection{Methods}

The laboratory tests were carried out by driving the cleaning device of the planar reciprocating vibrating screen as shown in Figure 16a and the cleaning device of the bionic screen as shown in Figure 16b through frequency converters (6ES6430-2UD27-1CA0, 6ES6430-2UD27-5CA0, Siemens, Germany). The bionic screen was divided into four areas of I, II, III, and IV with the same length along the longitudinal direction, and area $\mathrm{I}$ is the front of the bionic screen. The inlet airflow velocity of the bionic screen was $9.6 \mathrm{~m} / \mathrm{s}$, and its angle with the horizontal plane was $25^{\circ}$. The maximum concave depth was $50 \mathrm{~mm}$, and the rotational speeds of the cams were $90 \mathrm{r} / \mathrm{min}$. Compared with the planar reciprocating vibrating screen working under the optimal working parameters (the inlet airflow velocity was $9.6 \mathrm{~m} / \mathrm{s}$, and its angle with the horizontal plane was $25^{\circ}$, the amplitude was $19 \mathrm{~mm}$, and the vibration frequency was $5.15 \mathrm{~Hz}$ ). Maize mixture was fed through the oscillation grain pan driven by a vibrating motor, and the feeding mass of the oscillation grain pan could be adjusted by changing the exciting force of the vibrating motor. Before the test, the feeding mass was calibrated. The calibration results showed that the feeding mass of the oscillation grain pan was $5 \mathrm{~kg} / \mathrm{s}, 6 \mathrm{~kg} / \mathrm{s}$, and $7 \mathrm{~kg} / \mathrm{s}$ when the exciting force of the vibration motor was $1.2 \mathrm{kN}, 1.6$ $\mathrm{kN}$, and $2.3 \mathrm{kN}$, respectively.

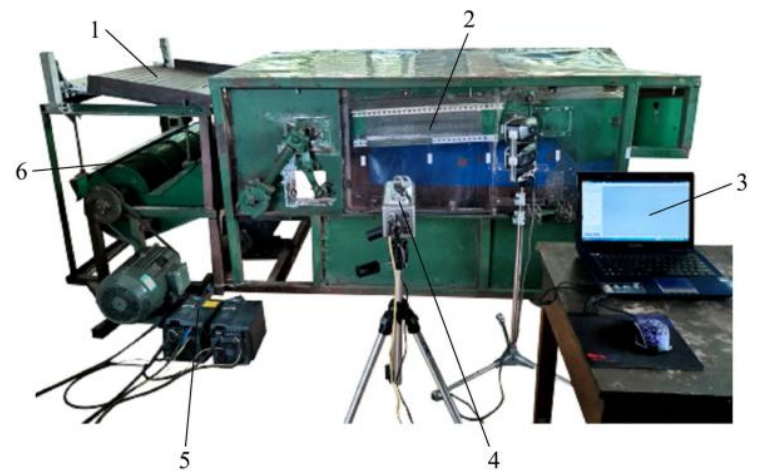

1. Oscillation grain pan 2. Planar reciprocating vibrating screen 3. Computer 4. High-speed camera 5. Frequency converter 6. Fan a. Cleaning device of planar reciprocating vibrating screen

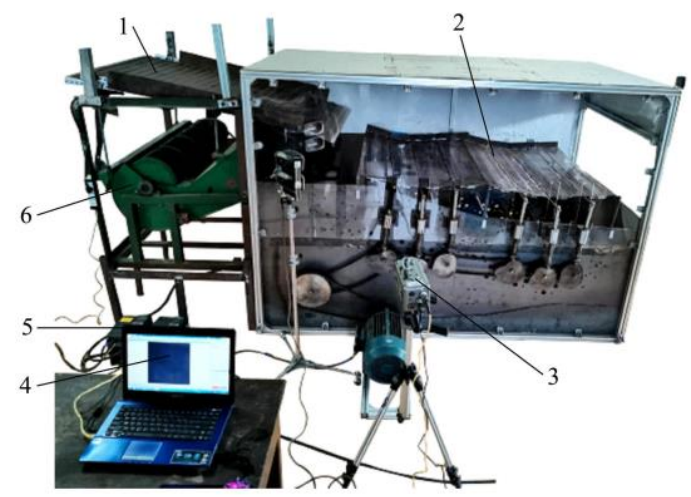

1. Oscillation grain pan 2. Bionic screen 3. High-speed camera 4. Computer 5. Frequency converter 6. Fan b. Cleaning device of bionic screen Figure 16 Test benches

The time of maize mixture migrated (TOMMM) in area I, the total screening time (TST), the loss percentage of maize grain (LPOMG), and the impurity percentage of maize grain (IPOMG) were the test indexes. Every test was repeated five times to obtain the mean value.

The TOMMM in area $\mathrm{I}\left(t_{m}\right)$ was calculated as follows:

$$
t_{m}=t_{2}-t_{1}
$$

where, $t_{1}$ is the time when maize mixture begins to appear in area I, $\mathrm{s} ; t_{2}$ is the time when all the maize mixture leaves area I, s.

The TST $\left(t_{s}\right)$ was calculated as follows:

$$
t_{s}=t_{3}-t_{1}
$$

where, $t_{3}$ is the time when all maize mixtures leave area IV, $\mathrm{s}$.

The LPOMG $\left(S_{L}\right)$ was calculated as follows:

$$
S_{L}=\frac{W_{L}}{W_{Z}} \times 100 \%
$$

where, $W_{L}$ is the mass of maize grains lost, $\mathrm{g} ; W_{Z}$ is the mass of all maize grains, g.

The IPOMG $\left(Z_{z}\right)$ was calculated as follows:

$$
Z_{z}=\frac{W_{z a}}{W_{h}} \times 100 \%
$$

where, $W_{z a}$ is the mass of impurities, $\mathrm{g} ; W_{h}$ is the total mass of maize grains and impurities under the screen, $g$.

\subsection{Results and analysis}

5.2.1 Analysis of the performance of maize mixture migrating of bionic screen

The test results of TOMMM in area I and TST of the planar reciprocating vibrating screen (PRVS) and the bionic screen (BS) with different feeding mass are shown in Figure 17. 

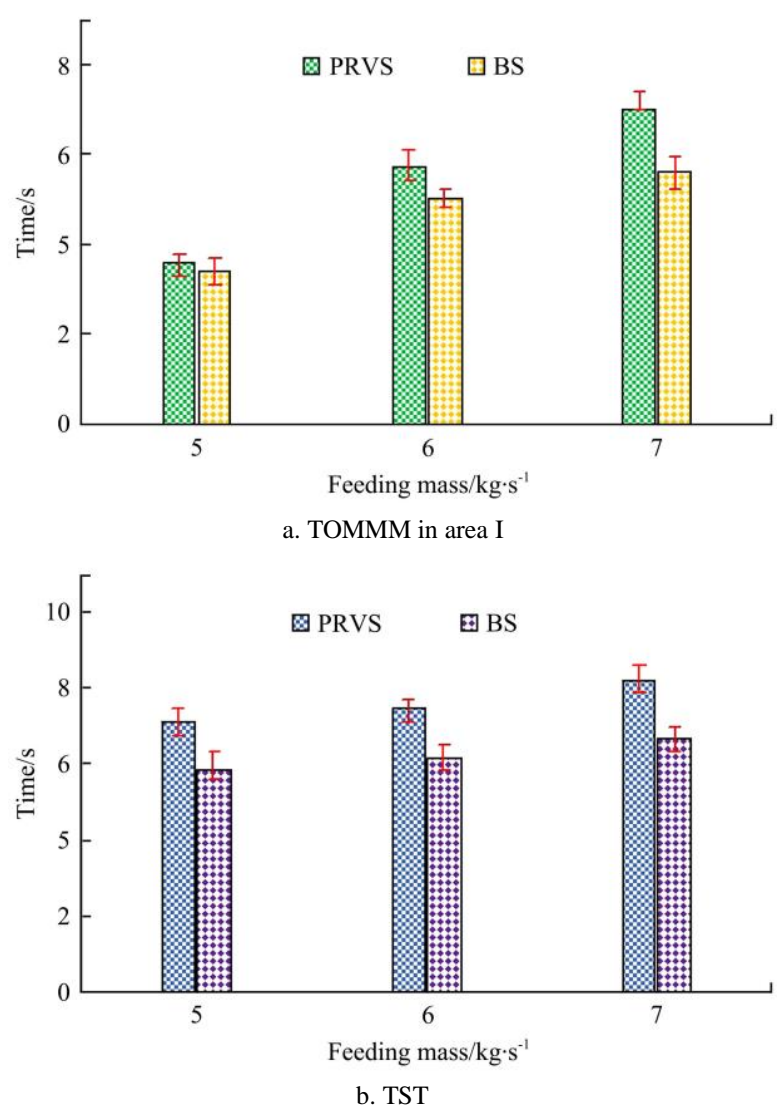

Figure 17 Performance of maize mixture migrated by planar reciprocating vibrating screen (PRVS) and the bionic screen (BS) with different feeding mass

Both TOMMM in area I and TST of the planar reciprocating vibrating screen and the bionic screen increase with the increasing feeding mass of the maize mixture. TOMMM in area I of the bionic screen was less than that of the planar reciprocating vibrating screen when the feeding mass was the same. When the feeding mass was $5 \mathrm{~kg} / \mathrm{s}, 6 \mathrm{~kg} / \mathrm{s}$, and $7 \mathrm{~kg} / \mathrm{s}$, TOMMM in area I of the bionic screen was $3.40 \mathrm{~s}, 5.03 \mathrm{~s}$, and $5.63 \mathrm{~s}$, respectively. Compared with the planar reciprocating vibrating screen, TOMMM in area I was shortened by $0.18 \mathrm{~s}, 0.71 \mathrm{~s}$, and $1.36 \mathrm{~s}$, respectively. It showed that the bionic screen had a stronger ability to migrate the maize mixture backward in the front of the screen when the feeding mass was the same. The average feeding mass of a 6-row maize grain combine harvester at normal operating velocity is $5 \mathrm{~kg} / \mathrm{s}$. With the increase of feeding mass, the advantage of migrating maize mixture backward in the front of the bionic screen became more and more apparent.

The phenomenon that maize mixture migrated backward rapidly in the front of the bionic screen could reduce the accumulation so that the maize mixture could continue to be migrated backward without hindering, which was beneficial to reduce the screening time of the maize mixture. The TST of the bionic screen was less than that of the planar reciprocating vibrating screen when the feeding mass was the same. When the feeding mass was $5 \mathrm{~kg} / \mathrm{s}, 6 \mathrm{~kg} / \mathrm{s}$, and $7 \mathrm{~kg} / \mathrm{s}$, TST of the bionic screen was $5.86 \mathrm{~s}, 6.14 \mathrm{~s}$, and $6.70 \mathrm{~s}$, respectively. Compared with the planar reciprocating vibrating screen, TST was shortened by $1.28 \mathrm{~s}, 1.33 \mathrm{~s}$, and $1.53 \mathrm{~s}$, respectively. Therefore, the screening efficiency of the bionic screen was higher than that of the planar reciprocating vibrating screen when the feeding mass was the same. The screening efficiency of the bionic screen was increased at a large feeding mass.
5.2.2 Analysis of screening performance of the bionic screen

The test results of LPOMG and IPOMG of the planar reciprocating vibrating screen and the bionic screen with different feeding mass are shown in Figure 18.
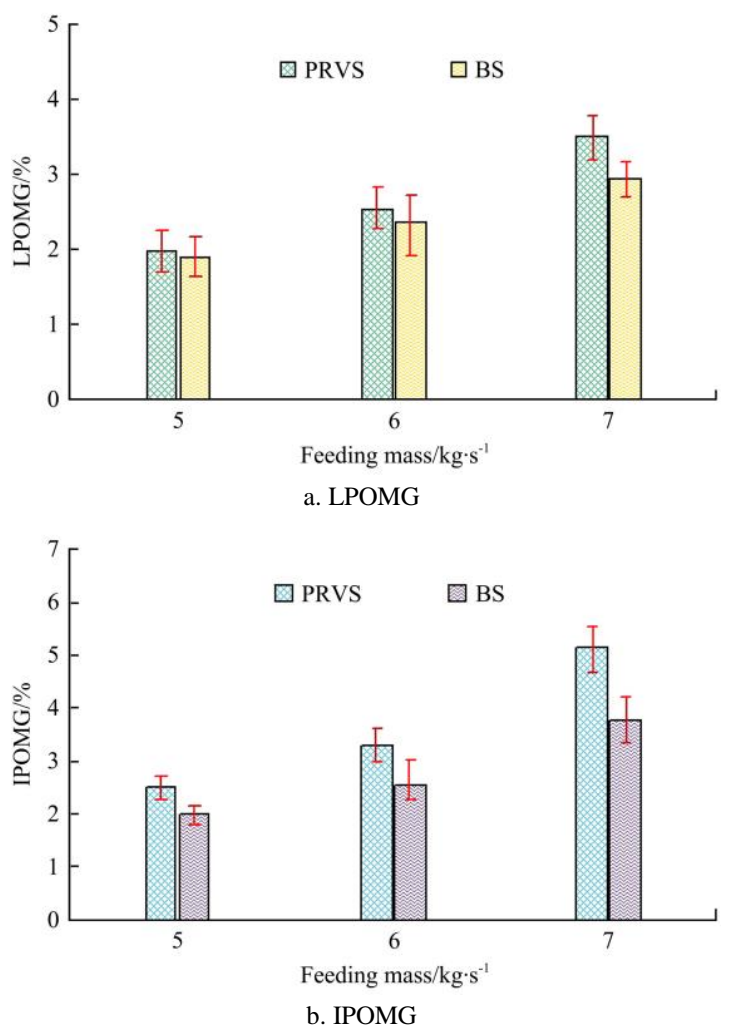

Figure 18 Screening performance of planar reciprocating vibrating screen (PRVS) and bionic screen (BS) with different feeding mass

Both LPOMG and IPOMG of the planar reciprocating vibrating screen and the bionic screen increase with the increasing feeding mass of maize mixture. The reason is that with the increase of feeding mass, the maize mixture mass to be screened per unit time increases, and the working load of the cleaning device becomes more extensive, resulting in the increase of the LPOMG. Simultaneously, the carrying effect of airflow on the impurities in the cleaning device is weakened, and the time for the impurities to be screened increases, resulting in the increase of the IPOMG after screening. When the feeding mass of maize mixture was set at 7 $\mathrm{kg} / \mathrm{s}$, compared with the planar reciprocating vibrating screen, the LPOMG and IPOMG of the bionic screen decreased by $0.54 \%$ and $1.40 \%$, respectively, which indicated that the bionic screen had better adaptability to large feeding mass. According to the data of the China Statistical Yearbook, the total maize output of Heilongiiang Province in 2019 was 39.398 million tons ${ }^{[31]}$. Compared with the planar reciprocating vibrating screen, the loss of maize grain in the cleaning process of the bionic screen could be reduced by 27.6-212.7 thousand tons, and substantial economic benefits could be created.

To determine the effects of feeding mass on TOMMM in area I, TST, LPOMG, and IPOMG, the data were analyzed using ANOVA, as shown in Table 3. Based on single-factor ANOVA ${ }^{[32]}$, it was founded that mean values of the TOMMM in area I, TST, LPOMG, and IPOMG differed significantly from each other $(p<0.05)$. According to Tukey's HSD test, the difference between the first and second groups for TST of the planar reciprocating vibrating screen and the bionic screen were not significant. The other mean values differed significantly from each other $(p<0.05)$. 
Table 3 ANOVA results for test indexes based on different feeding mass

\begin{tabular}{|c|c|c|c|c|c|c|c|c|c|}
\hline \multirow{2}{*}{ Screen } & \multirow{2}{*}{ Test index } & \multicolumn{5}{|c|}{ Single-factor ANOVA } & \multicolumn{3}{|c|}{ Tukey's HSD significance level ${ }^{[a]}$} \\
\hline & & Sum of squares & $d f$ & Mean square & $F$-value & $p$-value & $1-2$ & $1-3$ & $2-3$ \\
\hline \multirow{4}{*}{ PRVS } & TOMMM & 29.810 & 2 & 14.905 & 230.964 & 0.000 & 0.000 & 0.000 & 0.000 \\
\hline & TST & 3.121 & 2 & 1.561 & 22.826 & 0.000 & 0.153 & 0.000 & 0.002 \\
\hline & LPOMG & 5.929 & 2 & 2.965 & 55.359 & 0.000 & 0.006 & 0.000 & 0.000 \\
\hline & IPOMG & 18.087 & 2 & 9.044 & 118.495 & 0.000 & 0.001 & 0.000 & 0.000 \\
\hline \multirow{3}{*}{ BS } & TST & 1.811 & 2 & 0.905 & 11.425 & 0.002 & 0.294 & 0.001 & 0.022 \\
\hline & LPOMG & 2.795 & 2 & 1.398 & 21.756 & 0.000 & 0.037 & 0.000 & 0.007 \\
\hline & IPOMG & 7.902 & 2 & 3.951 & 43.760 & 0.000 & 0.032 & 0.000 & 0.000 \\
\hline
\end{tabular}

Note: ${ }^{\text {a] }}$ 1-2 indicates the significance level between group 1 (feeding mass $=5 \mathrm{~kg} / \mathrm{s}$ ) and group 2 (feeding mass $=6 \mathrm{~kg} / \mathrm{s}$ ), $1-3$ indicates the significance level between group 1 and group 3 (feeding mass $=7 \mathrm{~kg} / \mathrm{s}$ ), and 2-3 indicates the significance level between group 2 and group 3 .

\section{Discussion}

In further study, the simulation tests of the bionic screen will be conducted by the coupled method of computational fluid dynamics and discrete element method (CFD-DEM), and the parameters of the bionic screen cleaning device will be optimized. The maximum concave depth with the increase of the feeding mass and the effects of the installation angle of the bionic screen surface on the airflow field in the cleaning device will be explored.

The bionic screen is still in the test stage, and it will be further optimized to achieve optimal performance. To reduce the manufacturing cost of the bionic screen, its driving mechanism will be further optimized, or a new driving mechanism will be designed according to the motion characteristics of the bionic screen.

\section{Conclusions}

1) In this paper, earthworm was used as a bionic prototype. The contour curve of earthworm's head in an axial contracted state was extracted and fitted to obtain its equation. The maximum height of the earthworm's head somite in an axial contracted state was $8.99 \mathrm{~mm}$. The maximum height variation of earthworm's head somites was $3.48 \mathrm{~mm}$, and the maximum concave depth of the bionic screen was $50 \mathrm{~mm}$.

2) Based on the difference of the concave position of the wavy geometry of the lower surface during the earthworm moving, the motion of the bionic screen was divided into four postures. The cam drive mechanism was designed to realize the conversion between different postures of the bionic screen. The design of the cleaning screen based on the motion characteristics of the earthworm was completed.

3) The analysis of the screen plate motion showed that the requirements of the motion posture during the bionic screen moving could be realized by the cooperation between the cam drive mechanisms. The maize mixture to be migrated backward rapidly in the front of the bionic screen was realized.

4) When the feeding mass was the same, TOMMM in the front area and TST of the bionic screen were less than that of the planar reciprocating vibrating screen, and the difference between them further increased with the increase of feeding mass. The ability of maize mixture to be migrated backward in the front of the bionic screen was improved, which reduced the accumulation of the maize mixture and further improved the screening efficiency. When the feeding mass of the maize mixture was $7 \mathrm{~kg} / \mathrm{s}$, compared with the planar reciprocating vibrating screen, LPOMG and IPOMG of the bionic screen decreased by $0.54 \%$ and $1.40 \%$, respectively.

\section{Acknowledgements}

This work was supported by the National Natural Science Foundation of China (Grant No. 52075091, 51475090) and the Heilongiiang Provincial Natural Science Foundation of China (Grant No. E2017004).

\section{[References]}

[1] Yang L, Cui T, Qu Z, Li K H, Yin X W, Han D D, et al. Development and application of mechanized maize harvesters. Int J Agric \& Biol Eng, 2016; 9: 15-28.

[2] Liang Z W, Li Y M, De Baerdemaeker J, Xu L Z, Saeys W. Development and testing of a multi-duct cleaning device for tangential-longitudinal flow rice combine harvesters. Biosystems Engineering, 2019; 182: 95-106.

[3] Li H, Wang J S, Yuan J B, Yin W Q, Wang Z M, Qian Y Z. Analysis of threshed rice mixture separation through vibration screen using discrete element method. Int J Agric \& Biol Eng, 2017; 10(6): 231-239.

[4] Ma Z, Li Y M, Xu L Z. Discrete-element method simulation of agricultural particles' motion in variable-amplitude screen box. Computers and Electronics in Agriculture, 2015; 118: 92-99.

[5] Li J, Webb C, Pandiella S S, Campbell G M. Discrete particle motion on sieves-a numerical study using the DEM simulation. Powder Technology, 2003; 133: 190-202.

[6] Badretdinov I, Mudarisov S, Lukmanov R, Permyakov V, Ibragimov R, Nasyrov R. Mathematical modeling and research of the work of the grain combine harvester cleaning system. Computers and Electronics in Agriculture, 2019; 165: 104-966.

[7] Cui Z K, Di Z F, Zhou J, Zhang H, Bu L X, Gao Q. Design and research on 5TYS280 corn threshing and cleaning test bench. Journal of Agricultural Mechanization Research, 2017; 39: 113-117. (in Chinese)

[8] Dong K J, Yu A B. Numerical simulation of the particle flow and sieving behaviour on sieve bend/low head screen combination. Minerals Engineering, 2012; 31: 2-9.

[9] Zhao L L, Zhao Y M, Bao C Y, Hou Q F, Yu A B. Optimisation of a circularly vibrating screen based on DEM simulation and Taguchi orthogonal experimental design. Powder Technology, 2017; 310: 307-317.

[10] Ma Z, Li Y M, Xu L Z, Chen J, Zhao Z, Tang Z. Dispersion and migration of agricultural particles in a variable-amplitude screen box based on the discrete element method. Computers and Electronics in Agriculture, 2017; 142: 173-180.

[11] Liu C S, Wang H, Zhao Y M, Zhao L L, Dong H L. DEM simulation of particle flow on a single deck banana screen. International Journal of Mining Science and Technology, 2013; 23: 273-277.

[12] Deng J M, Shen H P, Li J, Wang X X, Huang T, He B X. Design and experiment for three-dimensional parallel kinematics vibration sieve. Transactions of the CSAM, 2013; 44: 342-346, 328. (in Chinese)

[13] Modrzewski R, Wodzinski P. Analysis of screening process of crushed basalt performed by a double-frequency screen. Physicochemical Problems of Mineral Processing, 2013; 49: 81-89.

[14] Jiang H S, Zhao Y M, Duan C L, Yang X L, Liu C S, Wu J D, et al. Kinematics of variable-amplitude screen and analysis of particle behavior during the process of coal screening. Powder Technology, 2017; 306: $88-95$. 
[15] Wang L J, Cui Y Q, Zheng Z H, Feng X, Shen B S, Li Y B. Effect of different motion forms of vibrating screen on screening of particle group. Transactions of the CSAM, 2019; 50: 119-129. (in Chinese)

[16] Ren L Q. Progress in the bionic study on anti-adhesion and resistance reduction of terrain machines. Science in China Series E: Technological Sciences, 2009; 52: 273-284.

[17] Zhao J L, Guo M Z, Lu Y, Huang D Y, Zhuang J. Design of bionic locust mouthparts stubble cutting device. Int J Agric \& Biol Eng, 2020; 13(1): 20-28.

[18] Ma Z, Li Y M, Xu L Z. Theoretical analysis of micro-vibration between a high moisture content rape stalk and a non-smooth surface of a reciprocating metal cleaning screen matrix. Biosystems Engineering, 2015; 129: 258-267.

[19] Cheng C, Fu J, Chen Z, Ren L Q. Design and experiment on modified sieve with coating of rice harvester. Transactions of the CSAM, 2020; 51: 94-102. (in Chinese)

[20] Trueman E R. The locomotion of soft-bodied animals. London: Edward Arnold, 1975; 200p.

[21] Quillin K M. Kinematic scaling of locomotion by hydrostatic animals: Ontogeny of peristaltic crawling by the earthworm Lumbricus terrestris. Journal of Experimental Biology, 1999; 202: 661-674.

[22] Liu G M. Coupling bionic research on the adhesion and resistance reduction of the earthworm surface. $\mathrm{PhD}$ dissertation. Jilin: Jilin University, 2009; 135p. (in Chinese)

[23] Jia H L, Li C Y, Zhang Z L, Wang G. Design of bionic saw blade for corn stalk cutting. Journal of Bionic Engineering, 2013; 10: 497-505.

[24] Tong J, Xu S, Chen D H, Li M. Design of a bionic blade for vegetable chopper. Journal of Bionic Engineering, 2017; 14: 163-171.

[25] Jia H L, Guo M Z, Zhao J L, Huang D Y, Zhuang J, Qi J T. Design and test of bionic wide-ridge soybean tilling-sowing machine. Int J Agric \& Biol Eng, 2019; 12(1): 42-51

[26] Wang Y, Li J Q, Zou M, Huang H, Xue L. Research on drag force for bionic cone soil contact part. Transactions of the CSAM, 2016; 47: 124-129. (in Chinese)

[27] Quaglia G, Nisi M. Design of a self-leveling cam mechanism for a stair climbing wheelchair. Mechanism and Machine Theory, 2017; 112: 84-104.

[28] Zhou W Q, Wang J W, Tang H. Structure optimization of cam executive component and analysis of precisely applying deep-fertilization liquid fertilizer. Int J Agric \& Biol Eng, 2019; 12(4): 104-109.

[29] Wang K Y. Metal heat treatment. Beijing: China water \& power Press, $2006 ; 252-253$

[30] Wei C C, Xu L Z, Wang J T, Li Y M. Inertial force balance and ADAMS simulation of the oscillating sieve and return pan of a rice combine harvester. Int J Agric \& Biol Eng, 2018; 11(1): 129-137.

[31] Statistics Bureau of the People's Republic of China. China statistical yearbook. Beijing: China Statistics Press, 2020.

[32] Gao X M, Xie H X, Gu F W, Wei H, Liu M J, Yan J C, et al. Optimization and experiment of key components in pneumatic peanut pod conveyor. Int J Agric \& Biol Eng, 2020; 13(3): 100-107. 